Pacific Journal of Mathematics

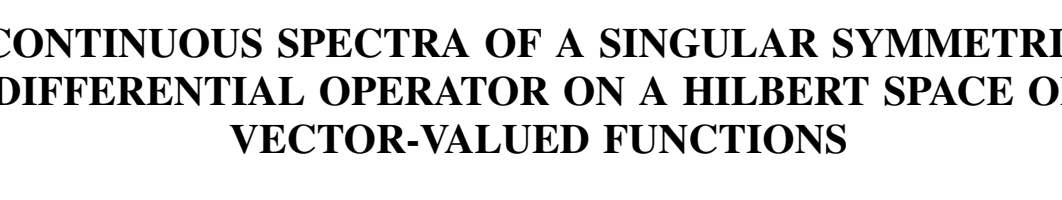




\section{CONTINUOUS SPECTRA OF A SINGULAR SYMMETRIC DIFFERENTIAL OPERATOR ON A HILBERT SPACE OF VECTOR-VALUED FUNCTIONS}

\section{ROBERT ANDERSON}

Let $H$ be the Hilbert space of complex vector-valued functions $f:[a, \infty) \rightarrow C^{2}$ such that $f$ is Lebesgue measurable on $[a, \infty)$ and $\int_{a}^{\infty} f^{*}(s) f(s) d s<\infty$. Consider the formally self adjoint expression $c(y)=-y^{\prime \prime}+P y$ on $[a, \infty)$, where $y$ is a 2-vector and $P$ is a $2 \times 2$ symmetric matrix of continuous real valued functions on $[a, \infty)$. Let $D$ be the linear manifold in $H$ defined by

\section{$D=\left\{f \varepsilon H: f, f^{\prime}\right.$ are absolutely continuous on compact subintervals of $[a, \infty), f$ has compact support interior to $[a, \infty)$ and $\iota(f) \varepsilon H\}$.}

Then the operator $L$ defined by $L(y)=\iota(y), y \varepsilon D$, is a real symmetric operator on $D$. Let $L_{0}$ be the minimal closed extension of $L$. For this class of minimal closed symmetric operators this paper determines sufficient conditions for the continuous spectrum of self adjoint extensions to be the entire real axis. Since the domain, $D_{0}$, of $L_{0}$ is dense in $H$, self adjoint extensions of $L_{0}$ do exist.

A general background for the theory of the operators discussed here is found in [1], [3], and [5]. The theorems in this paper are motivated by the theorems of Hinton [4] and Eastham and El-Deberky [2]. In [4], Hinton gives conditions on the coefficients in the scalar case to guarantee that the continuous spectrum of self adjoint extensions covers the entire real axis. Eastham and El-Deberky [2] study the general even order scalar operator.

DEFINITION 1. Let $\widetilde{L}$ denote a self adjoint extension of $L_{0}$. Then we define the continuous spectrum, $C(\widetilde{L})$, of $\widetilde{L}$ to be the set of all $\lambda$ for which there exists a sequence $\left\langle f_{n}\right\rangle$ in $D_{L}^{\sim}$, the domain of $\tilde{L}$, with the properties:

(i) $\left\|f_{n}\right\|=1$ for all $n$,

(ii) $\left\langle f_{n}\right\rangle$ contains no convergent subsequence (i.e., is not compact), and

(iii) $\left\|(\widetilde{L}-\lambda) f_{n}\right\| \rightarrow 0$ as $n \rightarrow \infty$.

For the self adjoint operator $\tilde{L}$ we have the following wellknown lemma.

LEMmA 1. The continuous spectrum of $\tilde{L}$ is a subset of the real numbers. 
Proof. Let $\lambda=\alpha+i \beta$ where $\beta \neq 0$. Then for all $f \in D_{L}^{-}$we can see by expanding $\|(\tilde{L}-\lambda) f\|^{2}$ that

$$
\|(\tilde{L}-\lambda) f\|^{2} \geqq|\beta|^{2}\|f\|^{2},
$$

which implies $\lambda \notin C(\widetilde{L})$.

THEOREM 2. Let $L(y)=y^{\prime \prime}+P(t) y$ for $a \leqq t<\infty$, where $P(t)=$ $\left[\begin{array}{ll}\alpha(t) & \gamma(t) \\ \gamma(t) & \beta(t)\end{array}\right]$ where $\gamma(t)$ is positive and has two continuous derivatives. Let $g(t)>0$ be one of $\alpha(t)$ or $\beta(t)$, where both $\alpha$ and $\beta$ are continuous on $[\alpha, \infty)$ and $g(t)$ has a continuous derivative. Then if for some sequence of intervals $\left\{A_{m}\right\}$ where $A_{m} \subseteq[a, \infty), A_{m}=$ $\left[c_{m}-a_{m}, c_{m}+a_{m}\right]$ and $a_{m} \rightarrow \infty$, the following are satisfied:

(i ) $\min _{x \in A_{m}}\{g(x)\} \rightarrow \infty$,

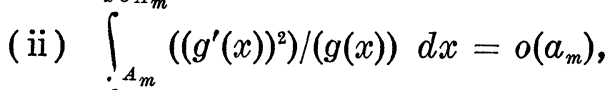

(iii) $\int_{A_{m}}^{A_{m}} g(x) d x=o\left(a_{m}^{3}\right)$,

(iv) $\int_{\Delta_{m}}[\gamma(x)]^{2} d x=o\left(a_{m}\right)$

we can conclude that $C(\tilde{L})$ is $(-\infty, \infty)$.

Proof. We will establish the theorem for $g(t)=\alpha(t)$ since the other case follows in exactly the same way.

Note that to prove the theorem then we need only show that for any real number $\mu$ there is a sequence $\left\langle f_{m}\right\rangle$ in $D(\widetilde{L})$ such that $\left\|f_{m}\right\|=1, f_{m} \rightarrow 0$ a.e., $f_{m}$ vanishes outside $A_{m}$ and $\left\|(\widetilde{L}-\mu) f_{m}\right\| \rightarrow 0$ as $m \rightarrow \infty$.

Let $\left\langle h_{m}\right\rangle$ be defined by

$$
h_{m}(t)=\left\{\begin{array}{lll}
{\left[1-\left\{\left(t-c_{m}\right) / a_{m}\right\}^{2}\right]^{3}} & \text { for } & \left|t-c_{m}\right| \leqq a_{m} \\
0 & \text { for } & \left|t-c_{m}\right|>a_{m}
\end{array}\right\} .
$$

Then define $\left\langle f_{m}(t)\right\rangle$ by

$$
f_{m}(t)=h_{m}(t)\left[\begin{array}{l}
b_{m 1} e^{i Q_{1}(t)} \\
b_{m 2} e^{i Q_{2}(t)}
\end{array}\right],
$$

where $Q_{1}, Q_{2}$ are real functions with two continuous derivatives and $b_{m 1}, b_{m 2}$ are normalization constants.

To find $\left|b_{m}\right|=\sqrt{b_{m 1}^{2}+b_{m 2}^{2}}$ we have

$$
\begin{aligned}
1 & =|| f_{m} \|^{2}=\int_{c_{m}-a_{m}}^{c_{m}+a_{m}}\left|b_{m}\right|^{2} h_{m}^{2}(t) d t=\left|b_{m}\right|^{2} \int_{-a_{m}}^{a_{m}}\left[1-\left(\frac{x}{a_{m}}\right)^{2}\right]^{6} d x \\
& =\left|b_{m}\right|^{2} \int_{-1}^{1} a_{m}\left[1-y^{2}\right]^{\mathrm{R}} d y=\left|b_{m}\right|^{2}\left(2 a_{m}\right)\left[1+\sum_{r=1}^{6}\left(\begin{array}{l}
6 \\
r
\end{array}\right)(2 r+1)^{-1}\right] .
\end{aligned}
$$


Hence for some positive constant $K$

$$
\left|b_{m}\right|^{2}=K\left(2 a_{m}\right)^{-1}
$$

and

$$
\left|f_{m}(t)\right| \leqq\left|b_{m}\right|=\sqrt{\bar{K}} / \sqrt{2} a_{m}
$$

Hence

$$
\begin{gathered}
f_{m} \rightarrow 0 \quad \text { as } \quad m \rightarrow \infty, \\
\left|h_{m}^{(r)}(t)\right| \leqq K_{r}\left(a_{m}\right)^{-r},
\end{gathered}
$$

where $K_{r}$ does not depend on $t$ or $m$.

Since $f_{m} \in D(\tilde{L})$, we have

$$
\begin{aligned}
(\widetilde{L}-\mu I) f_{m}= & f_{m}^{\prime \prime}+(P-\mu I) f_{m} \\
= & {\left[\begin{array}{l}
f_{m 1}^{\prime \prime}+(\alpha-\mu) f_{m 1}+\gamma f_{m 2} \\
f_{m 2}^{\prime \prime}+(\beta-\mu) f_{m 2}+\gamma f_{m 1}
\end{array}\right] } \\
(\widetilde{L}-\mu I) f_{m}= & {\left[\begin{array}{l}
\left\{-Q_{1}^{\prime 2}+(\alpha-\mu)\right\} f_{m 1}+\gamma f_{m 2}+i Q_{1}^{\prime \prime} f_{m 1} \\
\left\{-Q_{2}^{\prime 2}+(\beta-\mu)\right\} f_{m 2}+\gamma f_{m 1}+i Q_{2}^{\prime \prime} f_{m 2}
\end{array}\right] } \\
& +\left[\begin{array}{l}
b_{m 2} e^{i Q_{1}} h_{m}^{\prime \prime}+2 i Q_{1}^{\prime} b_{m 1} e^{i Q_{1}} h_{m}^{\prime} \\
b_{m 2} e^{i Q_{2}} h_{m}^{\prime \prime}+2 i Q_{2}^{\prime} b_{m 2} e^{i Q_{2}} h_{m}^{\prime}
\end{array}\right] .
\end{aligned}
$$

Now if $Q_{1}$ is chosen so that

$$
Q_{1}^{\prime 2}=\alpha-\mu, \quad Q_{1}^{\prime \prime}=\frac{\alpha^{\prime}}{2 \sqrt{\alpha-\mu}},
$$

and $b_{m 2}$ is chosen to be identically zero we have that

$$
(\tilde{L}-\mu I) f_{m}=\left[\begin{array}{l}
i Q_{1}^{\prime \prime} f_{m 1} \\
\gamma f_{m 1}
\end{array}\right]+\left[\begin{array}{l}
b_{m 1} e^{i Q_{1}} h_{m}^{\prime \prime}+2 i Q_{1}^{\prime} b_{m 1} e^{i Q_{1}} h_{m}^{\prime} \\
0
\end{array}\right] .
$$

By the way $Q_{1}$ is chosen,

$$
\left\|(\tilde{L}-\mu I) f_{m}\right\| \leqq\left\|\left(\frac{\alpha^{\prime}}{2 \sqrt{\alpha-\mu}}\right) f_{m}\right\|+\left\|\gamma f_{m}\right\|+\left\|b_{m} h_{m}^{\prime \prime}\right\|+\left\|2 Q_{1}^{\prime} b_{m} h_{m}^{\prime}\right\| .
$$

Now, by (ii)

$$
\left\|\frac{\alpha^{\prime}}{2 \sqrt{\alpha-\mu}} f_{m}\right\| \leqq\left[\frac{K}{a_{m}} \int_{A_{m}}\left(\frac{\alpha^{\prime}}{2 \sqrt{\alpha-\mu}}\right)^{2}\right]^{1 / 2}=o(1) \text { as } \quad m \longrightarrow \infty .
$$

By condition (iv),

$$
\left\|\gamma f_{m}\right\| \leqq\left(\frac{K}{a_{m}} \int_{A_{m}}|\gamma|^{2}\right)^{1 / 2}=o(1) \quad \text { as } \quad m \longrightarrow \infty
$$

Next, by (iii), (3) and (6) 


$$
\begin{aligned}
\left\|Q_{1}^{\prime} b_{m} h_{m}^{\prime}\right\| & =\left(\int_{A_{m}}(\alpha-\mu) \frac{K}{2 a_{m}} \cdot \frac{K_{1}^{2}}{a_{m}^{2}}\right)^{1 / 2} \\
& =K_{1} K^{1 / 2}\left(\frac{1}{2 \alpha_{m}^{3}} \int_{A_{m}}(\alpha-\mu)\right)^{1 / 2}=o(1) \text { as } m \longrightarrow \infty .
\end{aligned}
$$

Then, by (3), (6), and the Cauchy-Schwartz Inequality

$$
\begin{aligned}
\left\|b_{m} h_{m}^{\prime \prime}\right\| & \leqq\left(\int_{A_{m}}\left|b_{m}\right|^{2}\right)^{1 / 2}\left(\int_{A_{m}}\left|h_{m}^{\prime \prime}\right|^{2}\right)^{1 / 2} \\
& \leqq \sqrt{K / 2}\left(\int_{A_{m}}\left(K_{r}^{2} / a_{m}^{2}\right)\right)^{1 / 2}=o(1) \text { as } m \longrightarrow \infty
\end{aligned}
$$

Hence it follows that

$$
\left\|(\widetilde{L}-\mu I) f_{m}\right\| \longrightarrow 0 \text { as } m \longrightarrow \infty ，
$$

which is what we were to show.

Corollary 3. If $P(t)=\left[\begin{array}{ll}a t^{\sigma} & c t^{r} \\ c t^{r} & b t^{\beta}\end{array}\right]$ on some half-line $d \leqq t<\infty$ in Theorem 2 and

(i) $a, c>0$ with $\delta<0,0<\sigma<2$, or

(ii) $b, c>0$ with $\delta<0,0<\eta<2$ then $C(\widetilde{L})=(-\infty, \infty)$.

Theorem 4. Suppose $L(y)$ is as in Theorem 2, where $\gamma(t)$ is positive and has two continuous derivatives. If for some sequence of intervals $\left\{A_{m}\right\}$, where $A_{m}=\left[c_{m}-a_{m}, c_{m}+a_{m}\right], A_{m} \leqq[a, \infty)$ and $a_{m} \rightarrow \infty$, the following are satisfied:

(i) $\min _{t \in A_{m}}\{\gamma(t)\} \rightarrow \infty$,

(ii) $\int_{A_{m}}\left(\left(\gamma^{\prime}(t)\right)^{2}\right) /(\gamma(t)) d t=o\left(a_{m}\right)$,

(iii) $\int_{A_{m}} \gamma(t) d t=o\left(a_{m}^{3}\right)$,

(iv) $\int_{A_{m}} \alpha^{2}(t) d t$ and $\int_{A_{m}} \beta^{2}(t) d t$ are $o\left(a_{m}\right)$, then $C(\widetilde{L})=(-\infty, \infty)$.

Proof. In the proof of Theorem 2 choose $Q_{1}^{\prime 2}=Q_{2}^{\prime 2}=\gamma(t)-\mu$, so that $f_{m 1}=f_{m 2}$. Then $Q_{1}^{\prime \prime}=Q_{2}^{\prime \prime}=\left(\gamma^{\prime}(t)\right) /(2 \sqrt{\gamma(t)-\mu)}$ and applying conditions (i) - (iv) as before where $g(t)$ is replaced by $\gamma(t)$ we get that $\left\|(\widetilde{L}-\mu I) f_{m}\right\| \rightarrow 0$ as $m \rightarrow \infty$.

Corollary 5. Let $P(t)=\left[\begin{array}{ll}a t^{\sigma} & c t^{\tilde{\delta}} \\ c t^{\delta} & b t^{\eta}\end{array}\right]$ in Theorem 4. If $c>0$, $0<\delta<2$ and $\sigma, \eta<0$ then $C(\widetilde{L})=(-\infty, \infty)$. 
Let $H$ be the Hilbert space $\widetilde{L}_{2}([a, \infty), w)$ of complex vector-valued functions $f:[a, \infty) \rightarrow C^{2}$ such that $\|f\|^{2}=\int_{a}^{\infty} w\left(f^{*} f\right)<\infty$, where $w$ is positive and $w \in C^{(2)}[a, \infty)$. Let $l(y) \equiv(1 / w) y^{\prime \prime}+P y$. Then define $L_{0}$ as before and let $\widetilde{L}$ be a self adjoint extension of $L_{0}$.

THEOREM 6. Suppose there is a sequence of intervals, $A_{m} \subseteq$ $[a, \infty), A_{m}=\left[c_{m}-a_{m}, c_{m}+a_{m}\right]$ where $a_{m} \rightarrow \infty$ as $m \rightarrow \infty$, such that

(i) $\int_{A_{m}}\left(\alpha\left(w^{\prime}\right)^{2}\right) / w^{3}=o\left(\left|a_{m}\right|\right), \int_{A_{m}} \alpha / w=o\left(\left|A_{m}\right|\right)^{3}, \min _{t \in A_{m}} \alpha(t) \rightarrow \infty$,

$$
\int_{A_{m}}\left(w^{\prime}\right)^{4} / w^{6}=o\left(\left|A_{m}\right|\right), \int_{A_{m}}\left(w^{\prime \prime} / w^{2}\right)^{2}=o\left(\left|A_{m}\right|\right),
$$

$$
\int_{A_{m}} 1 / w^{2}=o\left(\left|A_{m}\right|^{5}\right)
$$

(iv) $\int_{A_{m}} \gamma^{2}=o\left(\left|A_{m}\right|\right)$

as $m \rightarrow \infty$. Then $C(\tilde{L})=(-\infty, \infty)$.

Note that (ii) implies that $\int_{A_{m}}\left(w^{\prime} / w^{2}\right)^{2}=o\left(\left|A_{m}\right|^{3}\right)$ by $\left(w^{\prime} / w^{2}\right)^{2}=$ $\left(w^{\prime}\right)^{2} / w^{3} \cdot 1 / w$ and Cauchy-Schwartz Inequality.

Proof. As is the previous theorem define

$$
f_{m}=\left[\begin{array}{l}
f_{m 1} \\
f_{m 2}
\end{array}\right] \text { where } f_{m 2}=0 \text { and } f_{m 1}=\left(b_{m} e^{i Q} h_{m}\right) w^{-1 / 2} .
$$

Then again $b_{m}^{2}=K / a_{m}$ and $\left|f_{m 1}\right| \leqq b_{m} w^{-1 / 2}=\left(K /\left(w a_{m}\right)\right)^{1 / 2}$. Calculating

$$
\begin{aligned}
f_{m 1}^{\prime}= & w^{-1 / 2} b_{m} e^{i Q} h_{m}^{\prime}+f_{m 1}\left[i Q^{\prime}-1 / 2 w^{-1} w^{\prime}\right] \\
f_{m 1}^{\prime \prime}= & f_{m 1}\left[-\left(Q^{\prime}\right)^{2}-i Q^{\prime} w^{-1} w^{\prime}+3 / 4 w^{-2}\left(w^{\prime}\right)^{2}-1 / 2 w^{-1} w^{\prime \prime}+i Q^{\prime \prime}\right] \\
& +b_{m} e^{i Q}\left[2 w^{-1 / 2} i Q^{\prime} h_{m}^{\prime}-w^{-3 / 2} w^{\prime} h_{m}^{\prime}+w^{-1 / 2} h_{m}^{\prime \prime}\right] .
\end{aligned}
$$

Then $(\widetilde{L}-\mu I) f_{m}=(1 / w) f_{m}^{\prime \prime}+P f_{m}$, where the top element is

$$
\begin{aligned}
& \frac{1}{w} f_{m 1}^{\prime \prime}+(\alpha-\mu) f_{m 1}=\frac{f_{m 1}}{w}\left[-\left(Q^{\prime}\right)^{2}+(\alpha-\mu) w\right] \\
& +\frac{f_{m 1}}{w}\left[-i Q^{\prime} w^{-1} w^{\prime}+\frac{3}{4} w^{-2}\left(w^{\prime}\right)^{2}-\frac{1}{2} w^{-1} w^{\prime \prime}+i Q^{\prime \prime}\right] \\
& +b_{m} e^{i Q}\left[w^{-3 / 2}\right]\left[2 i Q^{\prime} h_{m}^{\prime}-w^{-1} w^{\prime} h_{m}^{\prime}+h_{m}^{\prime \prime}\right] \\
& =\frac{f_{m 1}}{w}\left[-\left(Q^{\prime}\right)^{2}+(\alpha-\mu) w\right]+\frac{f_{m 1}}{w^{3}}\left[-i Q^{\prime} w w^{\prime}+\frac{3}{4}\left(w^{\prime}\right)^{2}-\frac{1}{2} w w^{\prime \prime}+w^{2} i Q^{\prime \prime}\right] \\
& +b_{m} e^{i Q} w^{-3 / 2}\left[2 i Q^{\prime} h_{m}^{\prime}-w^{-1} w^{\prime} h_{m}^{\prime}+h_{m}^{\prime \prime}\right] .
\end{aligned}
$$

Of course, the second element of $(L-\mu I) f_{m}$ is $\gamma f_{m 1}$. By choosing $\left(Q^{\prime}\right)^{2}=(\alpha-\mu) w$ we have that by (i) 


$$
\begin{aligned}
& Q^{\prime}=[(\alpha-\mu) w]^{1 / 2}=O\left((\alpha w)^{1 / 2}\right) \text { as } t \longrightarrow \infty . \\
& Q^{\prime \prime}=O\left(\frac{[\alpha w]^{\prime}}{\sqrt{\alpha w}}\right) \text { as } t \longrightarrow \infty .
\end{aligned}
$$

Then by the calculations above

$$
\begin{aligned}
\left\|(\tilde{L}-\mu I) f_{m}\right\| \leqq & \left\|\frac{f_{m 1}}{w^{2}} Q^{\prime} w^{\prime}\right\|+\frac{3}{4}\left\|\frac{f_{m 1}}{w^{3}}\left(w^{\prime}\right)^{2}\right\|+\frac{1}{2}\left\|f_{m 1} \frac{w^{\prime \prime}}{w^{2}}\right\| \\
& +\left\|\frac{f_{m 1} Q^{\prime \prime}}{w}\right\|+2\left\|b_{m} w^{-3 / 2} Q^{\prime} h_{m}^{\prime}\right\| \\
& +\left\|b_{m} w^{-5 / 2} w^{\prime} h_{m}^{\prime}\right\| \\
& +\left\|b_{m} w^{-3 / 2} h_{m}^{\prime \prime}\right\|+\left\|\gamma f_{m 1}\right\|
\end{aligned}
$$

Since $\left|f_{m 1}\right|^{2} \leqq K /\left(w a_{m}\right)$ and $\left(Q^{\prime}\right)^{2}=(\alpha-\mu) w$,

$$
\begin{aligned}
& \left\|f_{m 1} w^{-2} Q^{\prime} w^{\prime}\right\| \\
\leqq & \left(\frac{K}{a_{m}} \int_{A_{m}}(\alpha-\mu) w^{-3}\left(w^{\prime}\right)^{2}\right)^{1 / 2}=o(1) \quad \text { as } \quad m \longrightarrow \infty \quad \text { by (i) . }
\end{aligned}
$$

Similarly,

$$
\left\|f_{m 1} w^{-3}\left(w^{\prime}\right)^{2}\right\| \leqq\left(\frac{K}{a_{m}} \int_{A_{m}}\left[\left(w^{\prime}\right)^{2} w^{-3}\right]^{2}\right)^{1 / 2}=o(1) \quad \text { by (ii) }
$$

By the definition of $Q$ and $f_{m 1}$,

$$
\left\|f_{m 1} w^{-1} Q^{\prime \prime}\right\|=O\left(\int_{A_{m}} \frac{K\left[(\alpha w)^{\prime}\right]^{2}}{a_{m} \alpha w^{3}}\right)^{1 / 2}=o(1) \text { by (iii) . }
$$

And by condition (ii),

$$
\left\|f_{m 1} w^{-2} w^{\prime \prime}\right\| \leqq\left(\frac{K}{a_{m}} \int_{A m}\left[\left(w^{\prime \prime}\right)^{2} w^{-4}\right]\right)^{1 / 2}=o(1) .
$$

Since $\left|b_{m}\right|^{2}=K / a_{m}$ and $\left|h_{m}^{\prime}\right| \leqq K_{1} / a_{m}$,

$$
\left\|b_{m} w^{-3 / 2} Q^{\prime} h_{m}^{\prime}\right\| \leqq\left(\left(K K_{1}^{2} / a_{m}^{3}\right) \int_{A_{m}}\left(\frac{\alpha-\mu}{w}\right)\right)^{1 / 2}=o(1) \text { by (i) . }
$$

Similarly, by the remark at the end of the theorem,

$$
\left\|b_{m} w^{-5 / 2} w^{\prime} h_{m}^{\prime}\right\| \leqq\left(\left(K K_{1}^{2} / a_{m}^{3}\right) \int_{A_{m}}\left(w^{\prime}\right)^{2} w^{-4}\right)^{1 / 2}=o(1) .
$$

Since $\left|h_{m}^{\prime \prime}\right| \leqq K_{2} / a_{m}^{2}$,

$$
\left\|b_{m} w^{-3 / 2} h_{m}^{\prime \prime}\right\| \leqq\left(\left(K K_{2}^{2} / a_{m}^{5}\right) \int_{A_{m}} w^{-2}\right)^{1 / 2}=o(1) \quad \text { by (ii) . }
$$

By (iv), 


$$
\left\|\gamma f_{m 1}\right\| \leqq\left(\left(K / a_{m}\right) \int_{A_{m}} \gamma^{2}\right)^{1 / 2}=o(1) \text { as } m \longrightarrow \infty
$$

Hence, by the above calculations and (7),

$$
\left\|(\tilde{L}-\mu I) f_{m}\right\| \longrightarrow 0 \text { as } m \longrightarrow \infty \text {. }
$$

Since this is what we were to show, this conclude the proof.

\section{REFERENCES}

1. N. Dunford and J. T. Schwartz, Linear Operators, Part II: Spectral Theory, Interscience-Wiley, New York, 1963.

2. M.S.P. Eastham and A. A. El-Deberky, The Spectrum of differential operators with large coefficients, J. London Math. Soc., 2 (1970), 257-266.

3. I. M. Glazman, Direct Methods of Qualitative spectral Analysis of Singular Differential Operators, Israel Program for Scientific Translations, Jerusalem, 1965.

4. D. Hinton, Continuous spectra of second-order differential operators, Pacific J. Math., 33, No. 3 (1970), 641-643.

5. M. A. Naimark, Linear Differential Operators, Part II, Ungar, New York, 1968.

Received February 22, 1974, and in revised form October 8, 1974,

DeERing Milliken, InC. 



\section{PACIFIC JOURNAL OF MATHEMATICS}

EDITORS

RICHARD ARENS (Managing Editor)

University of California

Los Angeles, Calıfornia 90024

R. A. Beaumont

University of Washington

Seattle, Washington 98105
J. DugundJI

Department of Mathematics

University of Southern California

Los Angeles, California 90007

D. Gilbarg and J. Milgram

Stanford University

Stanford, California 94305

\section{ASSOCIATE EDITORS}
E. F. BECKENBACH
B. H. NeumanN
F. WOLF
K. YOSHIDA

\section{SUPPORTING INSTITUTIONS}

UNIVERSITY OF BRITISH COLUMBIA

CALIFORNIA INSTITUTE OF TECHNOLOGY

UNIVERSITY OF CALIFORNIA

MONTANA STATE UNIVERSITY

UNIVERSITY OF NEVADA

NEW MEXICO STATE UNIVERSITY

OREGON STATE UNIVERSITY

UNIVERSITY OF OREGON

OSAKA UNIVERSITY
UNIVERSITY OF SOUTHERN CALIFORNIA

STANFORD UNIVERSITY

UNIVERSITY OF TOKYO

UNIVERSITY OF UTAH

WASHINGTON STATE UNIVERSITY

UNIVERSITY OF WASHINGTON

AMERICAN MATHEMATICAL SOCIETY NAVAL WEAPONS CENTER 


\section{Pacific Journal of Mathematics}

\section{Vol. 55, No. $1 \quad$ September, 1974}

Robert Lee Anderson, Continuous spectra of a singular symmetric

differential operator on a Hilbert space of vector-valued functions . . . $\quad 1$

Michael James Cambern, The isometries of $L^{p}(X, K) \ldots \ldots \ldots \ldots \ldots . . \ldots$

R. H. Cameron and David Arne Storvick, Two related integrals over spaces of continuous functions ................................

Gary Theodore Chartrand and Albert David Polimeni, Ramsey theory and

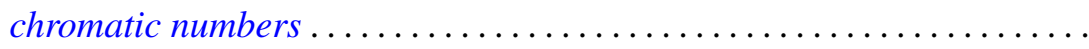

John Deryck De Pree and Harry Scott Klein, Characterization of collectively compact sets of linear operators ...................

John Deryck De Pree and Harry Scott Klein, Semi-groups and collectively compact sets of linear operators ....................... 55

George Epstein and Alfred Horn, Chain based lattices.............. 65

Paul Erdős and Ernst Gabor Straus, On the irrationality of certain series . . 85

Zdeněk Frolík, Measurable uniform spaces................... 93

Stephen Michael Gagola, Jr., Characters fully ramified over a normal

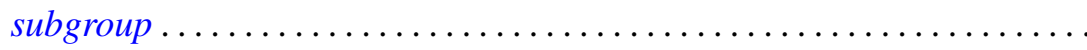

Frank Larkin Gilfeather, Operator valued roots of abelian analytic

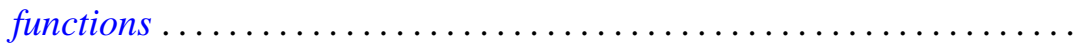

D. S. Goel, A. S. B. Holland, Cyril Nasim and B. N. Sahney, Best approximation by a saturation class of polynomial operators

James Secord Howland, Puiseux series for resonances at an embedded

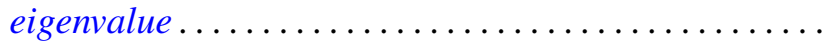

David Jacobson, Linear GCD equations .................

P. H. Karvellas, A note on compact semirings which are multiplicative

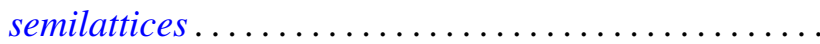

Allan Morton Krall, Stieltjes differential-boundary operators. II . .

D. G. Larman, On the inner aperture and intersections of convex sets

S. N. Mukhopadhyay, On the regularity of the $P^{n}$-integral and its application to summable trigonometric series ....... .

Dwight Webster Read, On $(J, M, m)$-extensions of Boolean algebras ....

David Francis Rearick, Multiplicativity-preserving arithmetic power series.

Indranand Sinha, Characteristic ideals in group algebras

Charles Thomas Tucker, II, Homomorphisms of Riesz spaces . . .

Kunio Yamagata, The exchange property and direct sums of indecomposable injective modules. 